\title{
Auto-Eficácia para Atividades Ocupacionais e Interesses Profissionais em Estudantes do Ensino Médio
}

\author{
Self-efficacy for occupational activities and \\ professional interests in students
}

Auto-eficacia para actividades ocupacionales e intereses profesionales en estudiantes de la enseñanza media
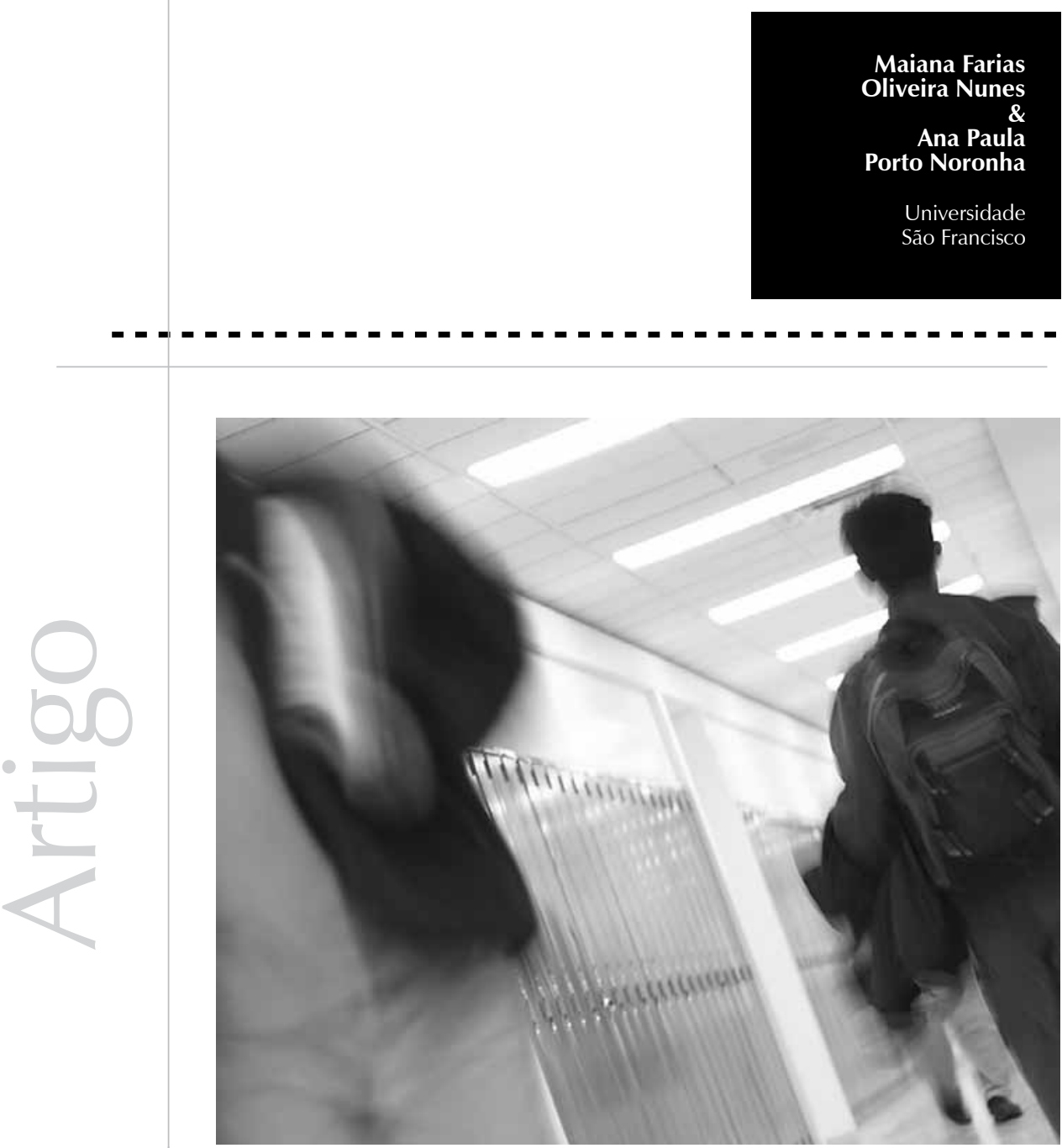
Resumo: A auto-eficácia para as atividades ocupacionais e os interesses profissionais descrevem, respectivamente, atividades que os jovens possuem confiança de que podem desempenhar e ações que gostariam de executar. Os objetivos deste artigo foram analisar diferenças de média na auto-eficácia e suas fontes e nos interesses em função das variáveis gênero, tipo de escola e série escolar. Participaram 333 alunos do ensino médio, com média de idade de 15,9 anos, sendo 182 mulheres, que responderam a EAAOc e o SDS, que avaliam auto-eficácia e interesses. A auto-eficácia, classificada de acordo com os tipos de Holland, apresentou padrão IRSAEC, e os interesses, ESIACR. Houve diferenças significativas em função do gênero para os interesses, a auto-eficácia e suas fontes. Não houve diferenças significativas para a auto-eficácia pela série escolar ou tipo de escola. Algumas fontes de auto-eficácia apresentaram diferenças pelo tipo de escola e série escolar, tendo o mesmo ocorrido com alguns perfis de interesse.

Palavras-chave: Auto-eficácia. Teoria social cognitiva. Interesses profissionais. Orientação profissional.

Abstract: Self-efficacy for occupational activities and professional interests are described, respectively, as the confidence of youngsters in the performance of certain job activities and actions that they would like to carry on. This paper aimed at analyzing the mean differences in self-efficacy, its sources and professional interests regarding gender, school type and scholar grade. 333 students from high school students took part in this research, mean age 15,9 years old, 182 female, who answered EAAOc and SDS that assess self-efficacy and interests. Self-efficacy, classified by Holland types, showed an IRSAEC pattern, while interests showed ESIACR. There were significant differences in self-efficacy, in its sources and in the interests that were related to gender. There were no differences for self-efficacy due to school type or scholar grade. Some sources of self-efficacy and some interest types showed mean differences due to scholar grade and type of school. Keywords: Self-efficacy. Social cognitive theory. Professional interests. Professional guidance.

Resumen: La auto-eficacia para las actividades ocupacionales y los intereses profesionales describen, respectivamente, actividades que los jóvenes poseen confianza de que pueden desempeñar y acciones que les gustaría ejecutar. Los objetivos de este artículo fueron analizar diferencias de media en la auto-eficacia y sus fuentes y en los intereses en función de las variables género, tipo de escuela y serie escolar. Participaron 333 alumnos de la enseñanza media, con media de edad de 15,9 años, siendo 182 mujeres, que contestaron a EAAOc y el SDS, que evalúan auto-eficacia e intereses. La auto-eficacia, clasificada de acuerdo con los tipos de Holland, presentó calidad IRSAEC, y los intereses, ESIACR. Hubo diferencias significativas en función del género para los intereses, la auto-eficacia y sus fuentes. No hubo diferencias significativas para la auto-eficacia por la serie escolar o tipo de escuela. Algunas fuentes de auto-eficacia presentaron diferencias por el tipo de escuela y serie escolar, habiendo ocurrido lo mismo con algunos perfiles de interés. Palabras clave: Auto-eficacia. Teoría social cognitiva. Intereses profesionales. Orientación profesional.

Um dos modelos teóricos propostos para estudar o desenvolvimento de carreira é um desdobramento da teoria sociocognitiva de Bandura (1986, 1997), denominado teoria sociocognitiva para o desenvolvimento de carreira. Nesse campo, há especial destaque para as formulações de Lent, Brown e Hackett (1994). Para os autores, existem alguns mecanismos sociocognitivos que influenciam o processo de escolha de uma profissão, e, entre eles, os mais importantes se referem às crenças de auto-eficácia e às expectativas de resultado. A auto-eficácia é compreendida como a confiança de uma pessoa em suas habilidades para realizar com sucesso uma tarefa ou um grupo de tarefas específicas, sendo uma variável que ajuda a explicar se um indivíduo terá iniciativa, perseverança e se conseguirá ter êxito em um determinado curso de ação. Já as expectativas de resultado são as crenças pessoais acerca dos possíveis resultados das ações, ou seja, as conseqüências imaginadas de certos atos.

A auto-eficácia e as expectativas de resultado, de acordo com essa perspectiva teórica, são mais importantes que a habilidade da pessoa em uma dada área, no momento da escolha de uma profissão. Em outras palavras, quando o sujeito possui habilidade em uma atividade, porém não possui crença 
de auto-eficácia e expectativa de resultado favorável quanto à mesma, provavelmente apresentará a tendência de não realizar escolhas profissionais nessa área (Lent et al., 1994).

Mais especialmente com relação às crenças de auto-eficácia, Bandura (1986) propõe que elas se originam de quatro fontes primárias, a saber, experiências pessoais, aprendizagem vicária, persuasão verbal e indicadores fisiológicos. A experiência pessoal, quando acompanhada por sucesso na execução da tarefa, leva a um aumento da crença de auto-eficácia, uma vez que as experiências funcionam como fontes autênticas para a crença na própria capacidade para realizar certas ações. Já a aprendizagem vicária ocorre por meio da observação do comportamento de outras pessoas, ou seja, quando uma pessoa considerada como "semelhante" na habilidade em questão (exemplo: leitura) possui um bom desempenho, isso deve elevar a crença de auto-eficácia do observador. O contrário tende a ocorrer quando pessoas semelhantes enfrentam fracassos sucessivos em dada atividade. A persuasão verbal ocorre por meio dos feedbacks sobre a o desempenho da pessoa em determinada tarefa, que podem encorajar ou desencorajar o sujeito. Por fim, os indicadores fisiológicos envolvem a observação e a interpretação de reações fisiológicas ao realizar certas ações, a partir das quais se pode concluir que a pessoa se encontrava, por exemplo, ansiosa ou com estado de ânimo positivo.

Outro construto considerado importante na escolha de uma profissão são os interesses profissionais, definidos por Lent et al. (1994) como padrões de gosto, aversão ou indiferença frente a certas atividades profissionais. Esses autores entendem que as crenças de auto-eficácia e as expectativas de resultado precedem a formação dos interesses. Mais especificamente, as percepções de eficácia e expectativa positiva acerca das recompensas futuras, por um lado, geram os interesses por atividades e estimulam intenções de continuar se comprometendo com elas. Por outro lado, aquelas situações vinculadas a crenças de eficácia e expectativas de resultado negativas provocam desgosto e evitação da atividade, gerando exclusões das opções de carreira ligadas a essas crenças.

Apesar da relação entre os conceitos de autoeficácia e interesses, os autores enfatizam que não se trata do mesmo construto (Lent et al., 1994; Lent, Brown, \& Hackett, 2004). Os interesses influenciam diretamente os planos de escolha, mas não serão necessariamente transformados em ação, pois outras variáveis estão envolvidas no processo. Sob essa visão, uma pessoa pode ter interesses por uma determinada profissão, porém, ao analisar as recompensas financeiras que poderia obter com ela, pode considerá-las insatisfatórias para o padrão que almeja, e assim, formular metas e engajar-se em ações que apontam outra direção. Os autores argumentam que o interesse sustentado ao longo do tempo por determinadas atividades é desenvolvido naqueles domínios nos quais as pessoas se consideram eficazes e nos quais antecipam resultados positivos. Já a auto-eficácia, por ser a crença de que se pode realizar com sucesso uma dada ação, deve preceder o desenvolvimento de interesses por uma dada atividade, marcando, por fim, a diferença entre os construtos.

Uma das formas para compreender os interesses é o modelo hexagonal, proposto por Holland (1963), que sugere seis tipos de perfis de interesse. Esses são denominados realista, investigativo, artístico, social, empreendedor e convencional, e são abreviados com a sigla RIASEC. O tipo realista destaca-se pelas habilidades mecânicas e atléticas, mas podem faltar habilidades sociais, pela valorização de elementos concretos ou de 
Quanto ao gênero,

Bandura (1986, 1997) sustenta que as crenças de autoeficácia são desenvolvidas de maneira diferenciada para homens e mulheres, em função de condições socioculturais, seja pelos modelos a que as pessoas tinham contato freqüente ou pelo ambiente social maior em que estavam inseridos. características pessoais tangíveis. Já o perfil investigativo tende a apresentar habilidades matemáticas e científicas mais desenvolvidas, mas normalmente faltam habilidades de liderança, com a valorização da ciência e a preferência por situações ou ocupações de pesquisa. O tipo artístico geralmente carece de habilidades burocráticas ou de organização, e tem a tendência de evitação das ocupações ou situações convencionais. Por outro lado, há uma aproximação com qualidades estéticas e elementos de arte. As pessoas do tipo social gostam de ajudar e de ensinar, e, por outro lado, tendem a ser desprovidas de habilidades mecânicas e científicas. O perfil empreendedor possui habilidades de liderança e de argumentação, mas há a tendência desse tipo à carência de habilidade científica. Por fim, o tipo convencional apresenta principalmente interesses burocráticos e aritméticos, carecendo de habilidades artísticas (Holland, Fritzsche, \& Powell, 1994). No Brasil, alguns estudos já foram conduzidos para estudar esse modelo de interesses, como é o caso das pesquisas de Mansão (2005), Negretti (2007), Primi, Moggi e Castellato (2004), Teixeira, Castro e Cavalheiro (2008), entre outros.

Outro aspecto que gera pesquisas e discussões teóricas são as diferenças observadas nas crenças de auto-eficácia e nos interesses no que diz respeito a características da amostra, tais como gênero, idade e nível socioeconômico. Quanto ao gênero, Bandura $(1986,1997)$ sustenta que as crenças de auto-eficácia são desenvolvidas de maneira diferenciada para homens e mulheres, em função de condições socioculturais, seja pelos modelos a que as pessoas tinham contato freqüente ou pelo ambiente social maior em que estavam inseridos. Complementando essa proposição, Lent et al. (1994) e posteriormente Lent et al. (2004) afirmam que os interesses profissionais também se expressam de maneira diferenciada para homens e mulheres.
Essas hipóteses teóricas foram trabalhadas em algumas pesquisas internacionais, como a de Lent, Lopez e Bieschke (1991), ao observarem que os interesses por matemática se desenvolviam de maneira diferenciada a depender do gênero. Mais especificamente, os homens apresentaram médias mais altas nessa área de interesse. A esse respeito, os autores acreditam que essa diferença se deve a maior exposição a temas relacionados, e sugerem que deve haver mais oportunidades para desenvolver interesse pela área.

Outro estudo também apontou resultados semelhantes. Lent, Lopez, Brown e Gore (1996), estudando as crenças de auto-eficácia e suas fontes relacionadas à matemática em alunos universitários, observaram a tendência de os homens reportarem maiores níveis de conquistas (experiência pessoal) relacionadas à matemática e percepções mais favoráveis dos indicadores fisiológicos que as mulheres. Em um segundo estudo, descrito nesse mesmo artigo, desta vez com alunos de ensino médio, foram encontrados resultados diferentes daqueles com universitários. Mais detalhadamente, as mulheres apresentaram mais experiências de modelação e persuasão que os homens, e não houve diferenças significativas para as experiências pessoais e para os indicadores fisiológicos. Como hipótese para os resultados, os autores sugerem que, no caso dos alunos mais jovens (ensino médio), talvez pelo nível de desenvolvimento em que se encontram, estes sejam mais sensíveis a informações providas de maneira diferenciada por pares etários e por adultos, com destaque para o papel das experiências de modelação e de persuasão verbal.

Ao estudar as fontes de auto-eficácia para habilidades sociais em universitários, Anderson e Betz (2001) observaram diferenças de gênero para os comportamentos sociais, sendo que as mulheres apresentaram médias mais elevadas em experiência pessoal, 
aprendizagem vicária e persuasão verbal. A investigação da auto-eficácia e suas fontes para a habilidade de escrita com alunos do ensino fundamental (ciclo I e II) e médio também indicou diferenças de gênero (Pajares \& Hobbes, 2005). Nessa pesquisa, as meninas obtiveram escores mais altos na experiência pessoal, aprendizagem vicária e persuasão verbal, além de baixa ansiedade. As meninas também obtiveram escores mais altos de auto-eficácia para a escrita, assim como foram mais bem avaliadas nesse domínio por seus professores. Os mais jovens (ciclo I do fundamental) apresentaram médias mais altas para as experiências pessoais de sucesso, aprendizagem vicária, persuasão verbal e auto-eficácia que os alunos do ciclo II (fundamental) e do ensino médio. Assim, além de diferenças por sexo, diferenças por estágios de desenvolvimento também têm sido investigadas.

Koumoundourou (2004) investigou os interesses profissionais e a auto-eficácia para tarefas ocupacionais específicas, ou seja, a confiança na habilidade para realizar tarefas específicas de carreiras, com relação a diferenças de gênero. Participaram estudantes gregos de ensino médio, sendo que os homens apresentaram médias significativamente mais altas que as mulheres na auto-eficácia para as habilidades lógicas, quantitativas, científicas e de negócios e nas habilidades que envolvem força física. Por outro lado, as mulheres tiveram escores mais elevados que os homens nas crenças de auto-eficácia para habilidades estéticas. Quanto aos interesses, avaliados por meio do SDS, baseado na tipologia de Holland, os homens tiveram resultados mais altos que as mulheres nos tipos realista; as mulheres obtiveram escores mais elevados nos tipos artístico e social.

Um estudo longitudinal foi conduzido para testar o efeito de interação ou mediação do sexo com relação às crenças de eficácia na escolha de graduações em matemática e ciências. Lapan, Shaughnessy e Boggs (1996) avaliaram estudantes universitários em dois momentos, sendo que a primeira medida ocorreu imediatamente após a conclusão do ensino médio e a segunda, três anos depois. O resultado mostrou que os homens demonstraram maior interesse por matemática e ciências e que estavam mais dispostos a escolher uma carreira associada ao tema que as mulheres. Ao dividir as carreiras em cinco áreas (artes, humanidades, ciências sociais, ciências biológicas e ciências físicas) e analisar a distribuição das carreiras escolhidas em função do gênero, verificou-se que as mulheres estavam mais alocadas nas ciências sociais, humanidades e artes ( $79 \%$ no primeiro momento e $85 \%$, no segundo) enquanto, do total de homens, $55 \%$ e $69 \%$ estavam nas mesmas três áreas, considerando os dois momentos, respectivamente.

Os resultados permitiram observar que as mulheres apresentaram diferenças significativamente menores que os homens quanto à quantidade de disciplinas cursadas relacionadas à matemática, tiveram escores menores nessa habilidade e possuíam autoeficácia mais baixa que os homens. Os autores discutem que as experiências anteriores vivenciadas pelos jovens de gêneros diferentes são qualitativamente diferentes, e que, no caso das mulheres, diminuem e restringem a gama de aspirações vocacionais no que diz respeito à matemática.

Outra investigação foi conduzida com um caráter longitudinal com mulheres, estudantes de ensino médio, que possuíam interesse por disciplinas consideradas não tradicionais para o seu gênero, ou seja, ciências, Matemática e engenharia (CME) para prever a escolha de cursos na área e auto-eficácia (Nauta \& Epperson, 2003). A média de idade na primeira coleta foi de 15,9, e, na segunda, de 20,4 anos. Nos dois momentos de coleta, observou-se que as alunas estavam mais interessadas em dados e idéias (perfil 
Em pesquisa realizada em Portugal, Almeida, Guisande, Soares e Saavedra (2006) revelam que o acesso ao ensino superior está muito vinculado às condições sociais da família de origem e ao valor sociocultural concedido

aos diferentes setores ou áreas profissionais. Os autores ressaltam que o jovem busca o que é mais "natural" dentro do grupo sociocultural a que se pertence, e assim se perpetua a condição

socioeconômica do grupo de origem. investigativo, na tipologia de Holland) que por coisas (perfil realista), sendo que ambos compõem o perfil geral das pessoas interessadas por CME.

Reardon, Bullock e Meyer (2005) conduziram um estudo que avaliou características relacionadas à tipologia de Holland com a força de trabalho americana entre as décadas de 1960 e 2000, com vistas a prover uma informação empírica sobre as escolhas das profissões, relacionadas com os interesses profissionais. Para a consecução das análises, os autores utilizaram um censo nacional que classifica as ocupações de acordo com o RIASEC. Quanto à década de 2000, que é mais útil para comparações com a presente pesquisa, as ocupações obtiveram percentuais, respectivamente entre tipos do RIASEC, de 43, 12, 2, 11, 22 e 10\%. No que tange às faixas salariais, as profissões ordenam-se de forma crescente, como CRAESI na década de 2000. As discrepâncias entre os tipos nesse aspecto são largas, sendo que o investigativo chega a ganhar, em média, o dobro do salário das profissões convencionais.

Essa pesquisa também investigou diferenças por gênero. Sob essa perspectiva, observouse uma estabilidade ao longo das décadas do realista para os homens e, para as mulheres, o tipo convencional foi o mais freqüente até a década de 1990, porém, em 2000, o tipo empreendedor recebeu destaque. De maneira mais ampla, pode-se afirmar que os homens estiveram, nas ocupações realista e empreendedora, entre $75 \%$ e $85 \%$ no período total, enquanto as mulheres estiveram em ocupações mais variadas ao longo das décadas, como a convencional, realista e social e, na década de 2000, também com maior participação no empreendedor. No que diz respeito à faixa etária e gênero dos trabalhadores, os homens mais jovens (1639 anos) estiveram nas profissões realistas (48\%) e empreendedoras (29\%), enquanto as mulheres da mesma faixa etária ocupavam cargos nos tipos empreendedor (30\%), convencional $(26 \%)$ e social $(22 \%)$.

As fontes de auto-eficácia associadas à tipologia de Holland foram estudadas em universitários por Williams e Subich (2006). Foram encontradas diferenças significativas em função do gênero para o realista e social $(p<0,001)$ e também para o investigativo $(p<0,01)$. Especificamente, mulheres reportaram menos experiências nos campos realista e investigativo, e os homens, menos experiências no tipo social. Os autores sugerem que experiências de aprendizagem diferenciais contribuem de maneira distinta para a auto-eficácia ocupacional e para as expectativas de resultado, limitando os interesses e os potenciais das carreiras consideradas.

Outro tema relevante refere-se às diferenças nas profissões escolhidas, que são associadas a fatores socioeconômicos. Em pesquisa realizada em Portugal, Almeida, Guisande, Soares e Saavedra (2006) revelam que o acesso ao ensino superior está muito vinculado às condições sociais da família de origem e ao valor sociocultural concedido aos diferentes setores ou áreas profissionais. Os autores ressaltam que o jovem busca o que é mais "natural" dentro do grupo sociocultural a que se pertence, e assim se perpetua a condição socioeconômica do grupo de origem. Considerando essa problemática, os autores analisaram o impacto do gênero e da origem sociocultural para o curso escolhido de estudantes da Universidade do Minho.

Nos resultados, tanto o gênero quanto a origem encontraram-se significativamente associados ao curso escolhido. Aproximadamente $61 \%$ dos homens freqüentava cursos de engenharia, e apenas 13,3\% ciências sociais. As mulheres predominam nos cursos de ciências sociais $(42,9 \%)$ e em menor proporção na engenharia $(19,2 \%)$. Os alunos de classe 
econômica menos favorecida freqüentam mais cursos de ciências sociais, enquanto os de classe mais favorecida freqüentam predominantemente os de engenharia. $\mathrm{Na}$ discussão, os autores confirmam sua expectativa teórica de diferenças de gênero na escolha de cursos, sendo que homens focam mais nas engenharias e mulheres nas ciências sociais, o que indica a importância dessa variável na escolha e na percepção de inserção profissional atrelada ao gênero. Em síntese, os autores alertam para o fato de que os de classe mais baixa tendem a escolher cursos de menor prestígio social (como as ciências sociais), enquanto alunos de classe mais alta escolhem preferencialmente cursos de engenharia e ciências naturais. De modo geral, observou-se uma tendência à reprodução de hierarquias sociais estabelecidas.

Outros construtos estudados em orientação profissional, como a cristalização das preferências profissionais, também indicam a existência de diferenças por tipo de escola, que pode ser associada ao nível socioeconômico (Balbinotti, Wiethaeuper, \& Barbosa, 2004). Ainda quanto a questões socioculturais associadas ao momento de escolha da profissão, pode-se destacar uma pesquisa, de natureza qualitativa, realizada sobre a escolha de especialidades médicas, que sugeriu que a escolha dessa profissão é influenciada pelos aspectos culturais, sociais e simbólicos dos participantes e de seus familiares (Fiore \& Yazigi, 2005). Desse modo, observa-se a importância da investigação de características da amostra como sexo, idade e nível socioeconômico para maior conhecimento das especificidades da mesma, que podem auxiliar na definição de intervenções e de pesquisas mais apropriadas.

Os objetivos da presente pesquisa referiramse à análise de diferenças na auto-eficácia e nos interesses profissionais em função das variáveis sexo, série escolar e tipo de escola.

\section{Método}

Participantes

A amostra se compôs de 333 estudantes, sendo 151 do sexo masculino e 182 do sexo feminino. Quanto às séries que os alunos cursavam, verificou-se que 154 estavam na primeira série do ensino médio, 104, na segunda série e 75 , na terceira. As idades variaram entre 14 e 19 anos, com média de 15,9 anos e desvio-padrão de 0,9 anos. Os alunos eram provenientes de quatro escolas do interior de São Paulo, sendo três particulares ( $n=111)$ e uma pública $(n=222)$.

Para as análises relacionadas aos interesses profissionais, apenas parte da amostra recémdescrita participou, mais especificamente, 289 alunos, sendo 114 da primeira série, 100, da segunda e 75, da terceira série, com idade média de 16 e desvio-padrão de 0,9 anos. No que se refere ao tipo de escola, 220 eram de escola pública e 69, de particular, e, quanto ao gênero, 133 participantes eram homens e 156, mulheres.

Instrumentos

Foi utilizada a Escala de Auto-eficácia para Atividades Ocupacionais-EAAOc (Nunes \& Noronha, no prelo). O EAAOc busca avaliar a auto-eficácia para atividades ocupacionais e as fontes de auto-eficácia associadas a elas. Compõe-se de duas seções, uma sobre a auto-eficácia para atividades ocupacionais e outra sobre as fontes de auto-eficácia. $\mathrm{Na}$ primeira, o participante indica três atividades que acredita poder realizar bem, associadas a alguma atividade do mundo do trabalho. As atividades listadas na primeira seção são fornecidas pelos próprios participantes, sem a apresentação de resposta dirigida. As respostas dos participantes são categorizadas de acordo com o referencial do RIASEC, que busca identificar a característica mais marcante do aspecto listado pelo sujeito. 
As fontes de auto-eficácia são avaliadas em 18 itens para cada atividade listada, variando de 1 (nenhuma influência) a 5 (muita influência) em seu nível percebido de influência. Os itens dessa seção avaliam as quatro fontes de auto-eficácia, porém, na análise fatorial, foram identificados três fatores, sendo eles a experiência pessoal direta (que engloba as experiências pessoais e os indicadores fisiológicos), a aprendizagem vicária (de pares ou de outras pessoas do convívio) e a persuasão verbal. As precisões dos fatores foram de $0,88,0,62$ e 0,72 , respectivamente (Nunes, 2007).

\section{SDS-Self-Directed Search Career Explorer (Holland et al., 1994)}

O SDS avalia os interesses profissionais por meio de seis tipos ocupacionais, que são o realista (R), o investigativo (I), o artístico (A), o social (S), o empreendedor (E) e o convencional (C), conhecidos pela denominação RIASEC, conforme indicado na introdução deste artigo. É composto por quatro seções, sendo que as três primeiras avaliam os seis tipos ocupacionais em termos de atividades, competências e carreiras, com 11 itens para cada tipo nas duas primeiras seções e 12 itens por tipo na terceira. Para essas três seções, as respostas possíveis são "sim" ou "não", e, na última, que trata das habilidades, o respondente deve se comparar com pessoas da mesma idade, com relação a 12 habilidades, que são medidas em uma escala likert de 7 pontos, de baixa a alta percepção de habilidade. A escala completa possui 216 itens, e os escores representam a soma dos escores parciais para cada tipo nas quatro seções.

No Brasil, já foram feitos alguns estudos que envolvem o SDS, sendo eles o de Primi et al. (2004), o de Mansão (2005), o de Mansão e Yoshida (2006) e, por fim, o de Sartori (2007). Os estudos, em conjunto, apontaram a utilidade do SDS para uso em jovens do ensino médio no Brasil. O manual do SDS, em versão traduzida e adaptada (Primi, Muniz, Mansão, \& Nunes, no prelo), encontra-se em processo de submissão ao Sistema de Avaliação dos Testes Psicológicos (SATEPSI), do Conselho Federal de Psicologia.

Procedimentos para a coleta de dados

Esta pesquisa foi submetida e aprovada por um Comitê de Ética em Pesquisa. Posteriormente, foi feito contato com instituições de ensino e com os responsáveis pelos jovens. Participaram da pesquisa apenas os estudantes que foram autorizados pelos pais ou responsáveis, por meio do Termo de Consentimento Livre e Esclarecido, para a colaboração na pesquisa. Os estudantes foram testados coletivamente em sala de aula, em uma única sessão, em horário previamente agendado com a instituição, havendo primeiro a aplicação do EAAOc e posteriormente do SDS, sendo que, em média, gastou-se 1 hora e meia com cada turma.

\section{Resultados}

O EAAOc teve suas duas seções analisadas separadamente, quanto à auto-eficácia para atividades ocupacionais e quanto às fontes associadas, enquanto o SDS teve o escore geral contemplado. Conforme os objetivos do artigo, foram realizadas análises de diferença de média e de freqüência em função das variáveis sexo, tipo de escola e série escolar.

Quanto à categorização das atividades listadas na primeira seção do EAAOc, houve uma freqüência total de 1001 respostas, e 764 foram aproveitadas. As demais respostas foram desconsideradas pelo fato de se referirem a conteúdos amplos ou ambíguos. A título de exemplo, o item "aprender a arrumar canos, motos, etc" foi categorizado como uma resposta do tipo realista; "entender sobre a 
origem da vida", como uma atividade investigativa; "fazer peças teatrais", como tipo artístico; "dar conselhos para as pessoas", como atividade social; "liderar", como empreendedor; "organizar meus objetos", como convencional. Vale destacar que se buscou categorizar as atividades listadas em apenas um dos tipos de Holland, aquele considerado mais representativo. No caso de atividades que possuíam uma definição ambígua, conforme já mencionado, esse dado não foi considerado para análise. A categorização foi realizada por uma das autoras do artigo, e, posteriormente, foram selecionadas aleatoriamente 100 respostas, que foram analisadas independentemente por dois estudantes de Psicologia, que classificaram os dados segundo as definições dos tipos de Holland. A classificação dos juízes foi comparada com a categorização da autora, que encontrou uma concordância de $92 \%$ entre a autora e o primeiro juiz, de $86 \%$ entre o segundo e a autora, e de $83 \%$ entre os dois juízes. Desse modo, considerou-se o processo de classificação confiável e foi possível dar continuidade às análises.

Entre os tipos do RIASEC, observaram-se freqüências de 222 atividades agrupadas no tipo realista; 225, no investigativo; 119, no artístico; 132, no social; 35, no empreendedor e 31, no convencional. Ressalta-se uma grande diferença na freqüência de auto-eficácia por profissões empreendedoras e convencionais, no cômputo geral. Assim, a auto-eficácia para atividades ocupacionais desse grupo pode ser descrita como IRSAEC. A análise de diferenças entre as distribuições das freqüências das atividades categorizadas foi feita por meio do Qui-quadrado, tendo sido observados índices significativos em função do gênero $\{\chi 2(5)=49,353, p<0,001\}$. Esse resultado é detalhado na Tabela 1.

Tabela 1. Freqüência da auto-eficácia para atividades ocupacionais categorizadas em função do gênero.

\begin{tabular}{llll}
\hline Tipologia & Masculino & Feminino & Total \\
\hline R & 122 & 100 & 222 \\
I & 90 & 135 & 225 \\
A & 52 & 67 & 119 \\
S & 25 & 107 & 132 \\
E & 9 & 26 & 35 \\
C & 10 & 21 & 31 \\
Quantidade de respostas (F) & 308 & 456 & 764 \\
\hline
\end{tabular}

Observou-se que homens indicaram maior auto-eficácia em atividades do tipo realista, e as mulheres, mais do investigativo e social. Uma análise mais qualitativa desse resultado se mostra pertinente. Observou-se maior percepção de auto-eficácia entre os homens em atividades que envolvem o contato com elementos da natureza, maior uso da força física, como no caso do tipo realista, e de atividades como estudar, pesquisar, ler, escrever e debater temas polêmicos no tipo investigativo. $\mathrm{O}$ mesmo se pode dizer para as atividades do tipo investigativo listadas pelas mulheres. No tipo social, foram incluídas respostas como aconselhamento, auxílio a pessoas com dificuldades, atividades de ensino e outras, caracterizando, assim, outro aspecto fortemente mencionado pelas mulheres. Vale realizar uma ressalva quanto a atividades realistas listadas pelas mulheres, que incluíram tarefas domésticas de limpeza e manutenção da casa, de modo distinto das respostas dos homens. 
Uma análise posterior envolveu possíveis diferenças na distribuição das freqüências das atividades em razão das séries escolares e do tipo de escola. Nesse aspecto, não foram encontradas diferenças significativas $\{\chi 2(10)=16,861, p=0,078\}$, tendo o mesmo ocorrido quando analisada a variável tipo de escola $\{\chi 2(5)=6,591, p=0,253\}$.

Outro tópico investigado nessa pesquisa foram as fontes de auto-eficácia. Quanto a possíveis diferenças de médias nas fontes de auto-eficácia no que tange à variável sexo, foi realizada a prova T de Student. Os fatores 1 e 3 apresentaram diferenças significativas $\{t(999)=-2,578, p=0,01 ; t$ $(1638)=-2,768, p=0,006$, respectivamente $\}$. Nos dois casos, as mulheres apresentaram médias mais elevadas, o que sugere maior influência das experiências pessoais diretas e da persuasão verbal para os jovens de sexo feminino.

No que diz respeito à análise das fontes pelo tipo de escola (pública e privada), houve diferenças significativas $\{t(999)=4,004, p<0,001\}$ apenas para o fator das experiências pessoais diretas, com uma média mais alta para a escola pública. Desse modo, no que se refere às atividades ocupacionais listadas pelos jovens, as experiências diretas provavelmente são vivenciadas com maior freqüência pelo jovem de escola pública. Já quanto à variável série escolar, procedeu-se à análise ANOVA, sendo que houve diferença significativa apenas para a aprendizagem vicária, $\operatorname{com} F(2,988)=2,240$ e $p=0,041$. Mais detalhadamente, as médias foram crescentes entre as segundas, primeiras e terceiras séries.

Quanto aos interesses estudados por meio do SDS, as médias foram observadas, de maneira decrescente, pela sigla ESIACR. Ao analisar diferenças no que diz respeito ao gênero, foram encontradas médias significativamente distintas nos tipos realista, social e convencional $\{t(288)=$ $9,550, p<0,001 ; t(288)=-7,487, p<0,001 ; t(288)=-1,933, p=0,054$, respectivamente $\}$. A Tabela 2 apresenta as médias e desvio-padrão para cada um dos tipos do SDS, em função do sexo.

Tabela 2. Média e desvio-padrão para os tipos do SDS divididos por sexo.

\begin{tabular}{llll}
\hline Tipo & Sexo & Média & Desvio-padrão \\
\hline Realista & Masculino & 21,3 & 9,53 \\
& Feminino & 11,9 & 7,25 \\
\multirow{3}{*}{ Investigativo } & Masculino & 23,3 & 10,17 \\
& Feminino & 24,6 & 10,46 \\
Artístico & Masculino & 20,5 & 12,23 \\
& Feminino & 22,1 & 11,66 \\
Social & Masculino & 19,8 & 10,10 \\
\multirow{2}{*}{ Empreendedor } & Feminino & 28,5 & 9,49 \\
& Masculino & 26,2 & 9,90 \\
Convencional & Feminino & 24,2 & 9,72 \\
& Masculino & 16,1 & 9,02 \\
& Feminino & 18,4 & 11,00
\end{tabular}

Considerando as médias nos tipos do SDS para homens, a sigla ficaria EIRASC, enquanto, para as mulheres, obteríamos SIEARC. Os homens obtiveram médias mais altas no perfil realista e as mulheres, no social e no convencional. As diferenças de média são mais visivelmente 
observadas nos tipos realista e social, porém não se pode negligenciar as diferenças no tipo convencional.

Já no que diz respeito a diferenças nos padrões de interesses em função das séries escolares, o tipo empreendedor e o convencional apresentaram médias significativamente diferentes ao longo das séries, na seqüência, $F(2,287)=3,131, p=$ 0,$04 ; F(2,287)=4,043, p=0,01$. Com o tipo empreendedor, as médias decresceram ao longo das séries, o que demonstra um padrão de diminuição dos interesses por essas ocupações com o passar do tempo. No tipo convencional, a média mais alta ocorre na $2^{\underline{a}}$ série, seguida de maneira decrescente pela $1^{a}$ e pela $3^{a}$ série. Contudo, esses dados devem ser interpretados com cautela, uma vez que podem indicar características específicas da amostra consultada. Por fim, no que diz respeito ao tipo de escola, não foram verificadas diferenças significativas em nenhum dos perfis do SDS.

\section{Discussão}

A presente pesquisa ganha relevância ao tratar de construtos amplamente pesquisados e discutidos, a saber, a auto-eficácia e os interesses profissionais, embora mais especialmente no exterior que no Brasil (Anderson \& Betz, 2001; Koumoundourou, 2004; Lent et al., 1991, 1996; Lapan et al., 1996; Nauta \& Epperson, 2003; Pajares \& Hobbes, 2005). Seu objetivo foi tratar a análise de diferenças de freqüência e de média na auto-eficácia para atividades ocupacionais e das fontes de auto-eficácia e dos interesses profissionais. Foram selecionados como parâmetros de comparação o sexo, o tipo de escola e a série escolar dos participantes. Os resultados são discutidos a seguir.

O resultado diferenciado da auto-eficácia em função do sexo parece parcialmente coerente com as observações de Reardon et al. (2005), no sentido de que os homens se encontram mais fortemente empregados em ocupações realistas e empreendedoras, enquanto as mulheres se distribuem, de maneira mais equilibrada, nos tipos convencional, realista, social e empreendedor, apesar de presentemente as mulheres terem se concentrado mais fortemente nos tipos social e empreendedor. Do mesmo modo, outras pesquisas já haviam evidenciado diferenças na auto-eficácia em campos ocupacionais específicos em função do gênero (Anderson \& Betz, 2001; Koumoundourou, 2004; Lent et al., 1991, 1996; Pajares \& Hobbes, 2005). No que respeita ao fato, reforça-se a hipótese de que práticas socioculturais e valores diferenciados podem gerar padrões de autoeficácia distintos em função do sexo.

Em síntese, este estudo reforçou pesquisas anteriores que apontaram diferenças de média na auto-eficácia associadas ao sexo, enquanto as séries escolares e o tipo de escola não geraram diferenças significativas. Podese levantar como hipótese, a esse respeito, que a confiança na própria capacidade para realizar tarefas ocupacionais esteve vinculada a práticas de socialização diferenciadas de homens e mulheres, ou seja, provavelmente, os pais, professores e outras pessoas estimularam os jovens de forma diferenciada a atividades ocupacionais. Nesse sentido, as asserções de Betz e Borgen (2000) são pertinentes na tentativa de transpor essa informação para a prática de aconselhamento ou orientação profissional/vocacional. Esses autores indicam que a auto-eficácia é um mediador do comportamento de escolha, que pode ser observado por meio de três indicadores, a saber, comportamentos de aproximação versus evitação, qualidade do desempenho no comportamento e persistência face a obstáculos ou experiências que não confirmam as crenças. Desse modo, o presente resultado ganha importância ao analisarmos as implicações para intervenções em orientação profissional/vocacional, uma 
vez que seria importante verificar se os padrões de auto-eficácia se encontram integrados à identidade vocacional de maneira harmoniosa. Em acréscimo, estudos futuros poderiam verificar se as opções vocacionais foram descartadas pelas práticas de socialização (comportamentos de evitação ou pouco estímulo para alcançar objetivos quando se depara com dificuldades), e que poderiam ser consideradas como opções viáveis de escolha vocacional.

No que diz respeito aos resultados diferenciados das fontes de auto-eficácia, em função do sexo, os resultados aproximamse dos de Anderson e Betz (2001) na pesquisa sobre as fontes de auto-eficácia para habilidades sociais, em que mulheres apresentaram médias mais elevadas em experiência pessoal de sucesso, aprendizagem vicária e persuasão verbal. O mesmo pode ser comentado quanto ao estudo de Pajares e Hobbes (2005) sobre as crenças de eficácia sobre a habilidade de escrita, no qual foi observada diferença de gênero, de tal modo que as meninas obtiveram escores mais altos na experiência de êxito, na aprendizagem vicária e na persuasão verbal, além de baixa ansiedade. No entanto, sugere-se que sejam realizadas outras pesquisas, com metodologias experimentais, que permitam estabelecer hipóteses mais específicas sobre o motivo de homens e mulheres receberem influência diferenciada no peso dado às fontes de auto-eficácia.

Já as diferenças observadas na fonte de auto-eficácia das experiências pessoais diretas quanto ao tipo de escola podem, em alguma medida, representar as origens socioculturais dos participantes e reiterar os resultados encontrados por Almeida et al. (2006), no sentido de que fatores associados à origem dos participantes influenciam nos elementos que envolvem a escolha profissional. No entanto, não parece pertinente tecer generalizações sobre essas diferenças encontradas, já que é necessária a realização de novos estudos que busquem corroborar ou refutar o atual resultado, uma vez que as características socioculturais da amostra não foram cuidadosamente controladas.

As séries escolares, por sua vez, apresentaram diferenças de média significativas na aprendizagem vicária. A esse respeito, cabem as considerações de Bandura (1997) no sentido de que as pessoas recebem influências com pesos variados de experiências diretas e indiretas (no caso da aprendizagem vicária) ao longo do tempo, sendo esperado que pessoas mais novas dêem mais importância às experiências indiretas como a aprendizagem vicária, uma vez que possuem pouca experiência direta pela pouca idade.

A análise dos interesses também apresentou resultados que devem ser discutidos. As diferenças em função do gênero aproximamse dos resultados internacionais encontrados por Reardon et al. (2005) no que diz respeito ao padrão de interesses de homens e mulheres. No entanto, há algumas divergências em relação aos resultados de Williams e Subich (2006), nos quais se verificaram diferenças significativas em função do gênero para o realista e o social e também para o investigativo, sendo que mulheres reportaram menos experiências nos campos realista e investigativo e os homens, menos experiências no tipo social. De maneira semelhante, Koumoundourou (2004) concluiu que os homens obtiveram resultados significativamente mais altos que as mulheres no tipo realista, porém as mulheres obtiveram escores mais elevados nos tipos artístico e social. Como possível hipótese para a discrepância observada entre o presente estudo e os demais, além da questão do sexo, é possível que a variável idade tenha um efeito de interação significativo, ou seja, talvez o gênero e idade devessem ser estudados conjuntamente, ao tratar da análise dos interesses. 
Ainda no que diz respeito aos interesses, a variável tipo de escola não esteve associada a diferenças significativas. Quanto à análise de diferenças de média por série nos interesses, apenas os tipos empreendedor e convencional obtiveram resultados significativamente distintos. No entanto, não houve um padrão comparável de diferenças entre o empreendedor e o convencional ao longo das séries. Especificamente no que diz respeito à diferença de média no tipo empreendedor, associada à série escolar, esse dado pode estar associado ao fato de os estudantes começarem a realizar mais atividades exploratórias quanto às profissões quando vão ficando mais velhos e diminuírem gradativamente o interesse pela área, talvez por motivos econômicos, uma vez que profissões empreendedoras talvez não sejam facilmente realizáveis no contexto brasileiro.

Por fim, uma comparação pertinente referese aos padrões de auto-eficácia e interesses demonstrados no presente estudo. A autoeficácia foi descrita pelo padrão decrescente IRSAEC, e os interesses, por ESIACR. Nesse item, esperava-se que os interesses fossem acompanhados por padrões semelhantes de auto-eficácia, o que, por sua vez, deveria levar a uma escolha profissional considerada satisfatória pelo sujeito (Lent et al., 1994, 2004). Considerando o perfil do grupo presentemente analisado em termos de interesses e auto-eficácia, observa-se maior discrepância no interesse e auto-eficácia realista e empreendedora, ou seja, os jovens possuíam maior confiança de que podiam realizar atividades ocupacionais realistas, mas não tinham interesse semelhante pela área. De forma semelhante, revelaram menor confiança na própria capacidade para atividades empreendedoras, porém possuíam grande interesse pela área. Esse resultado poderá ser melhor investigado por meio de análises que permitam esclarecer quais conseqüências/resultados os jovens imaginam que terão ao escolher profissões de tipos específicos, de modo a verificar a existência de mitos disfuncionais associados à escolha de profissões (por exemplo, é possível que os jovens imaginem que as profissões empreendedoras os tornarão ricos mais facilmente).

A presente pesquisa mostra-se, de maneira geral, em consonância com as observações de outros pesquisadores da auto-eficácia e dos interesses no âmbito vocacional, especialmente no que tange às diferenças por gênero. É necessária, no entanto, a realização de outras pesquisas, com amostras de outros Estados brasileiros e com idades mais variadas, de modo a detalhar as análises das influências desenvolvimentais e da origem sociocultural.

\section{Maiana Farias Oliveira Nunes}

Psicóloga, Mestre em Psicologia na área de Avaliação Psicológica pela USF, doutoranda em Psicologia pela USF. Bolsista Capes.

\section{Ana Paula Porto Noronha}

Doutora em Psicologia Ciência e Profissão pela Pontifícia Universidade Católica de Campinas. Docente do Programa de Pós-graduação Stricto Sensu em Psicologia da Universidade São Francisco.

\section{Endereço para envio de correspondência:}

Universidade São Francisco, Faculdade de Ciências Humanas. Rua Alexandre Rodrigues Barbosa, 45 - Centro.

Cep: 13251-900 - Itatiba, SP.

E-mails: maiananunes@mac.com / ana.noronha@saofrancisco.edu.br 
Referências
Almeida, L. S., Guisande, M. A., Soares, A. P., \& Saavedra, L. (2006). Acesso e sucesso no ensino superior em Portugal: questões de gênero, origem sociocultural e percurso académico dos alunos. Psicologia: Reflexão e Crítica, 19(3), 507-514.

Anderson, S. L., \& Betz, N. E. (2001). Sources of social selfefficacy expectations: Their measurement and relation to career development. Journal of Vocational Behavior, 58, 98-117.

Balbinotti, M. A. A., Wiethaeuper, D., \& Barbosa, M. L. L. (2004). Níveis de cristalização de preferências profissionais em alunos do ensino médio. Revista Brasileira de Orientação Profissional, 5(1), 15-28.

Bandura, A. (1986). Social foundations of thought and action: A social cognitive theory. Englewood Cliffs, NJ: Prentice-Hall.

Bandura, A. (1997). Self-efficacy: The exercise of control. New York: W. H. Freeman and Company.

Betz, N. E., \& Borgen, F. H. (2000). The future of career assessment: Integrating vocational interests with self-efficacy and personal styles. Journal of Career Assessment, 89(4), 329-338.

Fiore, M. L. M., \& Yazigi, L. (2005). Especialidades médias: estudo psicossocial. Psicologia: Reflexão e Crítica, 18(2), 200-206.

Holland, J. L. (1963). Explorations of a theory of vocational choice and achievement: A four-year prediction study. Psychological Reports, 12, 547-594.

Holland, J. L., Fritzsche, B. A., \& Powell, A. B. (1994). Sds-selfdirected search. Los Angeles, CA: Psychological Assessment Resources.

Koumoundourou, G. A. (2004). The reliability and validity of the Greek version of the Task-specific Occupational Selfefficacy Scale. British Journal of Guidance and Counseling, 32(1), 75-92.

Lapan, R. T., Shaughnessy, P., \& Boggs, K. (1996). Efficacy expectations and vocational interests as mediators between sex and choice of math/science college majors: A longitudinal study. Journal of Vocational Behavior, 49, 277-291.

Lent, R., Brown, S. D., \& Hackett, G. (1994). Toward a unifying social cognitive theory of career and academic interest, choice and performance. Journal of Vocational Behavior, 45, 79-122.

Lent, R., Hackett, G., \& Brown, S. D. (2004). Una perspectiva social cognitiva de la transición entre la escuela y el trabajo. Evaluar, 4, 1-22.

Lent, R., Lopez, F. G., \& Bieschke, K. J. (1991). Mathematics self-efficacy: Sources and relation to science-based career choice. Journal of Counseling Psychology, 38(4), 424-430.

Lent, R., Lopez F. G., Brown S. D., Gore, P. A., Jr. (1996). Latent structure of the sources of mathematics self-efficacy. Journal of Vocational Behavior, 49(3), 292-308.

Mansão, C. S. M. (2005). Interesses profissionais: validação do self-directed search career explorer-Sds. Tese de Doutorado, Pontifícia Universidade Católica de Campinas, Campinas, SP.

Mansão, C. S. M., \& Yoshida, E. M. P. (2006). SDS: Questionário de busca auto-dirigida - precisão e validade. Revista Brasileira de Orientação Profissional, 7(2), 67-80.

Nauta, M. M., \& Epperson, D. L. (2003). A longitudinal examination of the socialcognitive model applied to high school girls' choices of nontraditional college majors and aspirations. Journal of Counseling Psychology, 50(4), 448-457.

Negretti, F. (2007). Relação entre área de interesse e escolha profissional de estudantes de ensino médio. Trabalho de Conclusão de Curso, Universidade São Francisco, Itatiba, SP.

Nunes, M. F. O. (2007). Escala de fontes de eficácia percebida: aplicação com jovens em escolha profissional. Dissertação de Mestrado, Universidade São Francisco, Itatiba, SP.

Nunes, M. F. O., \& Noronha, A. P. P. (no prelo). Escala de autoeficácia para atividades ocupacionais - Eaaoc - manual técnico em preparação. São Paulo: Casa do Psicólogo.

Pajares, F., \& Hobbes, C. (2005). Sources of writing self-efficacy beliefs of elementary, middle and high school students. Trabalho apresentado no Meeting of the American Educational Research Association, Montreal, Canadá.

Primi, R., Moggi, M. A., \& Castelatto, E. O. (2004). Estudo correlacional do questionário de busca auto-dirigida (SDS) com o Ifp. Psicologia Escolar e Educacional, 8(1), 47-54.

Primi, R., Muniz, M., Mansão, C. M., \& Nunes, M. F. O. (no prelo). Sds-questionário de busca auto-dirigida-manual técnico da versão brasileira. São Paulo: Casa do Psicólogo.

Reardon, R. C., Bullock, E. E., \& Meyer, K. E. (2005). A holland perspective on the U.S. workforce from 1960 to 2000 technical report $n^{\circ} 45$. Tallahassee, FL: Center for the Study of Technology in Counseling and Career Development - The Florida State University.

Sartori, F. A. (2007). Estudo correlacional entre a escala de aconselhamento profissional (Eap) e o Sds. Dissertação de Mestrado, Universidade São Francisco, Itatiba, SP.

Teixeira, M. A. P., Castro, G. D., \& Cavalheiro, C. V. (2008). Escala de interesses vocacionais (EIV): construção, validade fatorial e consistência interna. Psicologia em Estudo, 13(1), 179-186.

Williams, M. C., \& Subich, C. M. (2006). The gendered nature of career related learning experiences: A social cognitive career theory perspective. Journal of Vocational Behavior, $69,262-275$. 